\title{
TENSIONES Y DILEMAS EN LOS PROGRAMAS DE PROXIMIDAD EN URUGUAY ${ }^{1}$
}

\section{TENSIONS AND DILEMMAS IN PROXIMITY PROGRAMS IN URUGUAY}

\section{Ximena Baráibar Ribero*}

\begin{abstract}
RESUMEN
El artículo se centra en los programas de proximidad que se implementaron en Uruguay desde el año 2012, en los cuales se plantea el trabajo de proximidad con los destinatarios como una de sus innovaciones. Con base en una investigación realizada en el 2016, en la cual se analizaron fuentes documentales y se realizaron 60 entrevistas a personas operadoras, supervisoras, facilitadoras y referentes temáticos. A partir de los resultados de estudio, se concluye que la forma de abordaje propuesta en los programas se encuentra poco fundamentada y colabora con procesos de individualización de la pobreza.
\end{abstract}

PALABRAS CLAVE: URUGUAY * POLÍTICA ASISTENCIAL * ESTADO * POBREZA * DESIGUALDAD

\section{ABSTRACT}

The article focuses on the proximity programs that have been implemented in Uruguay since 2012 and that considered proximity work with recipients as one of their innovations. Based on an investigation carried out in 2016, which involved the analysis of documentary sources and the conduct of 60 interviews, it is concluded that this form of approach is not well founded and that the concrete form that it adopts collaborates with processes of individualization of poverty.

KEYWORDS: URUGUAY * ASSISTANCE POLICY * STATE * POVERTY * INEQUALITY

1 Este artículo se basa en la investigación realizada por la autora del artículo en coautoría con Sandra Leopold, Carolina González y Laura Paulo, docentes e investigadoras del Departamento de Trabajo Social de la Facultad de Ciencias Sociales de la Universidad de la República, Uruguay.

* Departamento de Trabajo Social, Facultad de Ciencias Sociales, Universidad de la República, Montevideo, Uruguay. xbaraibar@gmail.com 


\section{INTRODUCCIÓN}

En el año 2005, el Frente Amplio asume el gobierno nacional en Uruguay. En un escenario de crisis social y económica, expresado en históricos niveles de pobreza, indigencia $y$ desocupación, comienza el desarrollo de la política asistencial como una de sus líneas fundamentales. Esto se expresa en innovaciones institucionales, básicamente en la creación del Ministerio de Desarrollo Social (MIDEs) y en un amplio conjunto de programas sociales en diversas arenas de la política social.

Desde el año 2012, en el segundo gobierno del Frente Amplio, se ponen en marcha diversos programas de proximidad, tales como: "Cercanías", "Jóvenes en Red" y "Uruguay Crece Contigo". Aunque dirigidos a públicos diferentes, tienen elementos comunes, en tanto, los tres definen un abordaje por un tiempo delimitado y territorial. Poseen baja materialidad e institucionalidad, sofisticados sistemas de registro $y$ monitoreo, $y$ se proponen vincular 0 revincular a los receptores con el mundo formal del trabajo y con el conjunto de los servicios sociales. Destacan como innovaciones la interinstitucionalidad de las propuestas y el trabajo de proximidad con la población destinataria.

Este artículo se enfoca en el segundo de los aspectos planteados como novedad. Específicamente se propone analizar y problematizar la forma en que el trabajo de proximidad es entendido (considerando el diseño de los programas, así como, la perspectiva de quienes trabajan en ellos), los fundamentos que lo sustentan y la forma que adopta esta estrategia durante el proceso de implementación.

Se trata de una discusión que se considera relevante, en tanto, los programas de proximidad se configuran en varios países de América Latina, como la última respuesta a la extrema pobreza y en el caso uruguayo, se han encontrado problemas para alcanzar los objetivos. Esta situación contribuye con algunos riesgos asociados a la política asistencial: enfocarse en la forma descuidando el contenido de la política pública y la responsabilidad a las personas en condición de pobreza de su situación y de las acciones para superarla.
Para alcanzar el objetivo planteado, el trabajo toma como base la investigación desarrollada durante el año 2016 titulada "Estudio de los dispositivos de proximidad en las estrategias de intervención de los programas Cercanías, Jóvenes en Red y Uruguay Crece Contigo del Ministerio de Desarrollo Social", que se sustenta en el análisis de la documentación de los programas y en 60 entrevistas realizadas a personas operadoras, supervisoras, facilitadoras y referentes temáticos ${ }^{2}$.

El artículo se organiza en tres partes. En la primera se ubican histórica y conceptualmente los programas de proximidad. Posteriormente, se enfatiza en la experiencia uruguaya, visualizando tanto el diseño como la implementación de los programas analizados. El artículo finaliza con un apartado de síntesis y conclusiones.

\section{POLÍTICA ASISTENCIAL Y PROGRAMAS DE PROXIMIDAD}

Los programas de proximidad constituyen la última de las innovaciones dentro de la política asistencial.

(...) las políticas que se dirigen a los pobres como sujetos, sabiendo que si bien operan incluso de manera más determinante sobre la situación de pobreza, otro conjunto importante de dispositivos institucionales, como por ejemplo las regulaciones laborales, la educación pública o el sistema jubilatorio, no son operatorias dirigidas a la pobreza, aunque modifiquen de manera determinante la distribución de la rique$z a$ en general $y$ en especial, sobre los pobres (Arias, 2012, p. 39).

2 La metodología utilizada supuso la realización de entrevistas semiestructuradas a integrantes de los programas en estudio. El material recolectado fue objeto de sistematización como de análisis a la luz de las categorías conceptuales que guiaron el proceso de investigación $y$ de otras construidas a partir del proceso de trabajo. Así, fue desarrollado un mapa conceptual reconstruyendo argumentos $y$ procedimientos que hacen parte de los ejercicios profesionales indagados y estableciendo las relaciones correspondientes. 
De acuerdo con Soldano y Andrenacci (2005) es a partir de los años 90, en el llamado Estado capitalista neoclásico, que se amplifica la política asistencial. Expresada en una red mínima de intervenciones sobre los grupos percibidos como portadores de mayor riesgo, se multiplicaron los programas sociales, con objetivos, plazos y financiamientos limitados. Esto se produce junto con una profunda reforma en las otras dos expresiones de la política social. En el caso del trabajo, se reforma el mercado garantista, orientándose a su desregulación y la reducción de los costos laborales. Se flexibilizan las pautas contractuales del fordismo y remercantiliza la cobertura de los riesgos, a través de los cuasimercados de gestión privada. Esta orientación también tuvo impactos en la política de servicios universales (educación, salud e infraestructura), implicando la revisión de las competencias públicas.

Filgueira (2015) destaca un posterior momento, configurado como un modelo abierto $y$ de orientación exportadora, pero con participación renovada del Estado. Señala algunas esferas de la política social: jubilaciones, pensiones $y$ otras transferencias monetarias para adultos mayores y familias con hijos. También, el acceso al aseguramiento y a los servicios de salud, así como cambios en la oferta y la edad cubiertos por la educación. Finalmente, indica políticas de derechos laborales y la protección a la persona trabajadora.

En similar sentido, Martínez Franzoni y Sánchez-Ancochea (2016) señalan a partir del año 2000, un nuevo momento que en el marco de "un giro a la izquierda", al menos en parte, revierte el período anterior. Las transformaciones giraron en torno a tres componentes que supusieron un mayor acceso a beneficios sociales en tanto derechos ciudadanos. A las medidas indicadas por el primer autor, agregan la preocupación por un "cuarto pilar" del bienestar, asociado a los cuidados. Todo esto ha supuesto el pasaje de coberturas restringidas a otras más amplias, pero de carácter segmentado.

Ese momento supone que el Estado retoma su actuación en la política de trabajo y en los servicios universales, pero a diferencia del período posterior a la Segunda Guerra Mundial, mantiene y expande la política asistencial. Dentro de esta, destacan los Programas de Transferencias Condicionadas (PTC) surgidos a finales de la década de los 90. De acuerdo a Cecchini y Atuesta (2017), estos otorgan transferencias monetarias a hogares pobres $y$ bajo ciertas condiciones que buscan mejorar las capacidades humanas. Procuran disminuir la pobreza a corto plazo, sosteniendo niveles básicos de consumo y a largo plazo, a través de mejoras en la salud y la educación de niños, niñas y adolescentes de esos hogares.

En el financiamiento $y$ en la promoción de los PTC (como en los anteriores Fondos de Inversión Social) tienen gran relevancia el Banco Mundial y el Banco Interamericano de Desarro1lo. Esto mismo ocurre - particularmente con el último de los organismos mencionadosen los programas de proximidad, presentados como la última innovación en la política asistencial. El argumento central a favor de estos surge de la constatación de que las poblaciones más vulnerables no acceden a los servicios sociales $y$ de dos ideas, que desde esta perspectiva se asocian a la pobreza: su multidimensionalidad y su expresión en diversas particularidades.

Dentro del primer aspecto de ese argumento, especial referencia reciben los PTC. Los promotores de los programas de proximidad reconocen su uso como incentivo para mejorar las condiciones de vida de las familias económicamente pobres y el ser una herramienta para desarrollar su capital humano. Pese a esto, se indica que es preciso definir nuevas medidas más sensibles a los determinantes de la vulnerabilidad y exclusión. En relación al segundo aspecto, se señala que nuevas concepciones sobre la pobreza la definen como multidimensional (no restringida solamente al ingreso) y esto exige diseñar intervenciones integrales $y$ que no sean respuestas estandarizadas (Jara, Barriga, Villafuerte y González-Acero, 2013; Jara y Sorio, 2013).

En una preocupante simplificación, las respuestas a lo indicado se colocan, casi exclusivamente, en el trabajo de proximidad y la articulación de los servicios. Aún cuando se haga referencia a la cobertura de estos servicios, no es donde se focaliza la atención, por tanto, no 
hay un debate sobre las estructuras productoras de desigualdad. En cambio se indica que los modelos más tradicionales de oferta de servicios, sectoriales, parecen no producir los efectos esperados (Jara et ál., 2013) y en otro documento se afirma que esta nueva modalidad de abordaje se basa en la necesidad de suplementar ciertos servicios para llegar a poblaciones que por alguna razón, carecen de cobertura (Jara y Sorio, 2013). Los elementos que explicarían estas constataciones, no aparecen y surgen afirmaciones que no problematizan los aspectos que las generan.

A lo señalado, los promotores de estos nuevos programas agregan lo que consideran un elemento esencial para mejorar las condiciones de vida de las poblaciones en mayor desventaja: la arquitectura institucional de las políticas. Una débil institucionalidad limita la posibilidad de ir a las poblaciones y lograr mejor impacto (Jara et ál., 2013). Nuevamente, el análisis opera por fuera de la desigualdad $y$, en cambio, se traslada a la forma de la prestación de servicios y no a su contenido y relevancia.

Jara y Sorio (2013) indican la existencia de determinantes críticos de la condición de pobreza; pero no es en este aspecto que se centra la atención, sino en la desigual distribución e intensidad de esos factores que dificultan la generación de paquetes cerrados de prestaciones. Por su parte, Jara et ál. (2013) destacan la necesidad de diagnósticos e intervenciones individualizadas y adaptadas a cada realidad.

Entonces las respuestas a la pobreza extrema se ubican en el trabajo articulado para generar o impulsar redes de atención social y se agrega que el centro de ese debate está en la necesidad de estrategias integrales, diversas, más próximas a la demanda y un vínculo más efectivo con los servicios sociales (Jara et ál., 2013). Parecerían estar aquí los determinantes de esa realidad. Finalmente, Jara y Sorio (2013) señalan que el acompañamiento familiar supone:

(...) un tipo de consejería, altamente estructurada, que involucra procesos de largo plazo y se orienta a metas vinculadas a modificar prácticas para mejorar la seguridad y el bienestar de las personas.
Su tecnología principal es la presencia de un profesional o educador especialmente entrenado para brindar apoyo psicosocial familiar o personal (p. 3).

\section{POLÍTICA ASISTENCIAL Y PROGRAMAS DE PROXIMIDAD EN URUGUAY}

En 2012, comienzan a implementarse los programas de proximidad ("Cercanías", "Jóvenes en Red" y "Uruguay Crece Contigo") constituyendo la última innovación en la política asistencial. Esta línea de actuación pública toma impulso en el país a partir de 2005, cuando el Frente Amplio asume el gobierno nacional. En este año, es creado el Ministerio de Desarrollo Social (MIDES) y dispositivos de carácter central y territorial, orientados a la coordinación y a promover la cercanía territorial, la descentralización y la participación.

Asimismo, se desarrolla un amplio conjunto de programas sociales: primero, entre 2005 y 2007, en el marco del Plan de Atención a la Emergencia Social (PANES); posteriormente, desde el 2008 dentro del Plan de Equidad; finalmente, a partir de 2011, dentro de la Reforma Social. De acuerdo a un estudio realizado por el Instituto de Ciencia Política de la Facultad de Ciencias Sociales de la Universidad de la República, entre 2005 y 2009, son creados 132 programas, estando el $72 \%$ dirigidos a la pobreza (MIDEs, 2010). Luego de este estudio y en el marco del Observatorio Social, el MIDEs continúa el relevamiento de la oferta pública social.

Del trabajo realizado por el Observatorio surgen dos mapeos de la matriz de protección social. En el relevamiento efectuado entre 2011-2012, resulta que son 358 los programas sociales desarrollados por organismos públicos de carácter nacional; de ellos, 51 se dirigen a situaciones de especial vulneración de derechos y 146 se orientan a la pobreza (MIDES, s.f. a). El segundo relevamiento, efectuado entre 2014-2015, ajusta la forma de organización de los programas. De los 330 existentes en ese momento, 155 se dirigen a la pobreza, mientras que los restantes 12 , están conformados por componentes universales $y$ focalizados (MIDES, s.f. b). Como puede observarse, aunque se produce 
una reducción de los programas implementados, el peso relativo de los dirigidos a la pobreza y vulnerabilidad aumenta, pasando del 40,7\% de los programas relevados en 2011 y 2012, al $47 \%$ unos años después.

Pese a este amplio y diversificado conjunto de intervenciones, comienzan a implementarse los programas "Cercanías", "Jóvenes en Red" y "Uruguay Crece Contigo", que plantean como una de sus innovaciones, el trabajo de proximidad con los destinatarios. Este abordaje se configura en una dimensión relevante por dos motivos: es uno de los ejes con los que se espera atender a la pobreza extrema; además, debido a que la interinstitucionalidad (que constituye el otro componente planteado como innovador) es débil o ausente, los equipos realizan el trabajo con los sujetos básicamente cara a cara, en su domicilio o entorno cercano.

\section{PROXIMIDAD: LA CONCEPTUALIZACIÓN DE LOS PROGRAMAS}

Aún cuando los tres programas tengan distintos desarrollos, el trabajo de proximidad aparece en cada uno de sus diseños. Esto surge tanto en sus líneas de actuación como en su estructura organizativa. En el programa "Cercanías", se declara que supone un esfuerzo interinstitucional para la atención de familias en situación de extrema vulnerabilidad. En su documento fundacional señala cuatro lineamientos conceptuales: la interinstitucionalidad, la integralidad, el trabajo con familias en clave territorial y la proximidad. En relación a esta última se indica que:

(...) supone asumir la intervención cercana a la familia, acompañando sus procesos, las urgencias, necesidades, preferentemente en el domicilio o en los espacios donde transita, orientando en la perspectiva de fortalecer sus capacidades y autonomía, no sustituyendo a la familia (MIDES, INAU, ANEP, ASSE, MVOTMA, 2012).

Posteriormente, se indica que la orientación señalada no desconoce las desventajas que el domicilio pueda tener para el trabajo de ciertas temáticas, recomendándose en ese caso espacios institucionales y comunitarios (Cercanías, 2015). Este último documento explicita que el trabajo de proximidad supone posibilitar el acceso a recursos y servicios, tal y como surgía en el texto fundacional. Se señala que:

El Abordaje de proximidad propuesto por la Estrategia son acciones sostenidas para fortalecer $y$ desarrollar capacidades y autonomía de la familia en el marco del ejercicio de derechos. El mismo implica el acceso a servicios y prestaciones del Estado así como la movilización de recursos personales, familiares y comunitarios (Cercanías, 2015, p. 16).

La proximidad es también observable en la estructura organizativa del programa, integrada por una comisión político-institucional, una Unidad de Gestión, espacios locales y regionales de articulación de servicios y los Equipos Territoriales de Atención Familiar (ETAF). Estos son señalados como el componente innovador del programa, trabajando en forma integral con metodologías de proximidad. Están integrados por técnicos en Trabajo Social, Psicología, Educación Social y otras disciplinas sociales y humanas (MIDES, INAU, et ál., 2012).

El programa "Jóvenes en Red" se dirige a adolescentes y jóvenes de 14 a 24 años, que no estudian ni culminaron el ciclo básico, no tienen empleo formal $y$ pertenecen a hogares con ingresos por debajo de la línea de pobreza (MIDES, MEC, MTSS, MDN, MDYT, ANEP/UTU, INAU, 2012a). Es el programa que menor desarrollo realiza sobre el trabajo de proximidad en su diseño, lo cual es observable en dos aspectos: en el apelo a lo territorial y comunitario, así como en su estructura operativa. En relación al primer aspecto, se señala como objetivo general "promover el ejercicio de derechos de esa población, desde un abordaje integral, territorial y en comunidad" (MIDES, MEC, et ál., 2012, p. 8). Más adelante, en el mismo documento, se indica que ese abordaje posibilitará adecuar el programa a las demandas y características de la población de cada territorio y localidad.

El Programa se desarrolla con base en cuatro componentes (1.Convocatoria, apoyo, 
acompañamiento $y$ seguimiento para el egreso; 2) Espacios de formación; 3) Laboral; 4) Proyección y fortalecimiento comunitario), de los cuales interesa destacar el primero, que también da indicios del trabajo de proximidad. Este refiere a la convocatoria, apoyo, acompañamiento a los jóvenes $y$ al seguimiento para el egreso. La convocatoria se realiza en cada territorio donde se lleva adelante el programa y el acompañamiento supone un acuerdo educativo, trabajo con familias, trabajo comunitario y con instituciones locales.

En relación a la estructura operativa de "Jóvenes en Red", esta se conforma por el Equipo de Gestión y el de Trabajo Territorial, con la tarea de desarrollar los cuatro componentes mencionados. Estos equipos están integrados por un coordinador, docentes/educadores, talleristas, profesional técnico con formación en comunidad, grupos o instituciones, así como personal de servicio $y$ administrativo (MIDEs, MEC, et ál., 2012).

Con un desarrollo intermedio de lo que supone el trabajo de proximidad, se ubica "Uruguay Crece Contigo" (UCC) que se dirige a hogares con mujeres embarazadas, niños y niñas menores de 4 años, priorizando aquellas situaciones que presenten vulnerabilidad social o sanitaria y se estructura con base en cuatro componentes. El primero refiere a la generación de conocimiento, el segundo a las acciones socio-educativas-sanitarias, el tercero al acompañamiento familiar $y$ trabajo de cercanía, y el último, a la orientación para promover el fortalecimiento de capacidades institucionales (UCC, s.f.a).

En relación al tercer componente, se señala que incluye las acciones socioeducativas con las familias, las cuales se realizan a través de equipos de cercanías. Principalmente, esto supone trabajo de orientación en el hogar y de diagnóstico en los temas vinculados a los objetivos del programa, así como, la mejora de la accesibilidad a prestaciones $y$ servicios (ucc, s.f. b).

El trabajo de proximidad es visualizable a partir de la estructura organizativa. ucc está dirigido por una Mesa de Coordinación Interinstitucional y la implementación es gestionada por una Unidad Técnica Central y facilitadores regionales. Para el componente de acompañamiento familiar y trabajo de cercanía, el programa cuenta con supervisores y equipos de cercanía. Estos últimos, integrados por técnicos del área social y de la salud, tienen a su cargo un conjunto de familias en un territorio de referencia para dar apoyo y seguimiento (UCC, s.f. a).

Como puede observarse, el trabajo de proximidad aparece bajamente conceptualizado y menos aún, fundamentado. En sintonía con lo que fuera presentado al inicio, aparece vinculado al acceso a las prestaciones y servicios. Los motivos que hacen que sea necesario un dispositivo de este tipo, en un contexto de ampliación de la intervención del Estado y de existencia de dispositivos centrales $y$ territoriales enfocados en la coordinación, no aparecen en la información analizada. Tampoco existe una problematización sobre las condiciones de vida de los receptores de estos programas, ni del entramado institucional con el cual aspiran a vincularlos.

En el relato de las personas entrevistadas surgen algunas cuestiones relevantes. En primer lugar, de manera similar a la documentación analizada, es baja la conceptualización en torno a lo que constituye el trabajo de proximidad y básicamente se observa una descripción de lo que se realiza. De modo que la proximidad se asocia fuertemente a la salida de las instituciones, a partir del desplazamiento al lugar donde la población destinataria se encuentra y a componentes de índole afectivo.

Lo que se entiende de proximidad es un acompañamiento fuerte $y$ cercano, se basa en que el joven no siempre llega a las instituciones sino que llegamos nosotros, una vez que se empieza a trabajar con el joven la idea es que ellos de a poco vayan llegando (Operador Jóvenes en Red, comunicación personal, 10/03/2016).

La característica innovadora de lo que es ETAF es la proximidad con las familias en territorio, en su medio cotidiano, en la casa de las familias, también en las instituciones que las familias se vinculan en su medio y su contexto (...) va la intervención técnica a la casa o los 
lugares que las familias circula y necesita que estemos (Operadora Cercanías, comunicación personal, 22/04/2016).

En los programas "Cercanías" y ucc, la referencia básica es el hogar, aunque en ambos se describe un proceso que supone no considerar exclusivamente ese espacio, pudiendo ser también una institución, una plaza, etc. En el programa "Jóvenes en Red", la referencia fundamental es el espacio público, debido a las características y lugares de encuentro de la población a la que se dirigen.

Cercanía era porque ibas a la casa, nada más. Este concepto se fue construyendo, podes generar cercanía sin estar en la casa, si bien implica esa llegada, es estar en contacto, establecer una referencia, acompañar, estar disponible y generar un vínculo de confianza (Supervisora Ucc, comunicación personal, 28/03/2016).

Además de asociada a la proximidad física, esta forma de trabajo también lo está a componentes afectivos $y$ vinculares. Esto parecería ser un aspecto ineludible, diferenciador $y$ es destacado como muy valorado por los receptores de los programas.

Dado que vengo a cumplir un lugar de referente frente al joven lo tengo que hacer desde un vínculo con él o ella. Es en ese sentido que es la estrategia más potente, la construcción de un vínculo donde se ponga en juego lo afectivo (Coordinador Jóvenes en Red, comunicación personal, 09/03/2016).

Para mí es construir vínculo, como el punto cero $y$ desde donde se para uno siempre. Porque si hago tal cosa puede ir en desmedro del vínculo o si hago tal otra puede favorecer. $\mathrm{O}$ si le entrego o no le entrego. El vínculo es como la vedette del asunto (Operadora Cercanías, comunicación personal, 03/03/2016).
De acuerdo con lo anterior, para las personas entrevistadas, la proximidad está fundamentada en poder acceder a sujetos a los cuales el resto de las instituciones no llega. Esta explicación convive con el dato de que una gran parte de quienes participan de los programas de proximidad vienen derivados de otros programas; incluso, en varios casos, de otros a los cuales se aspira a vincular a partir de esta particular experiencia. Resulta extraño que ambos aspectos no sean vinculados en las entrevistas.

[La proximidad] tiene que ver con poder llegar a una cantidad de jóvenes que no estaban ni estudiando ni trabajando y que además estaban por debajo de la línea de pobreza, es como un núcleo fuerte que estaba casi desafiliado de todo (Operador Jóvenes en Red, comunicación personal, 10/03/2016).

[El trabajo] está basado en la cercanía y en la capacidad de trabajar desde el hogar cosas que de repente en otros lados no se pueden. La idea es llegar a personas que están un poco alejadas de otros entornos, como por ejemplo, CAIF, policlínicas, entre otros (Operadora UCC, comunicación personal, 30/03/2016).

A partir de esa llegada, el trabajo se orienta a vincular o revincular con esas instituciones o con el mercado formal de trabajo, pero en la práctica el trabajo está más ligado con los sujetos que con los ámbitos a los cuales se espera que accedan $y$ permanezcan.

Se intenta mejorar lo que había antes, el tratar de que las familias más vulneradas accedan a servicios $y$ a prestaciones $y$ el trabajo de Cercanías acompaña ese intento de que las familias accedan a sus derechos vulnerados (Operadora Cercanías, comunicación personal, 22/04/2016).

Muchos de estos gurises no terminaron la escuela o arrancaron el liceo y el sistema los expulsó, entonces hay que 
volver a reinsertarlos con toda esa historia negativa que tuvieron (Operador Jóvenes en Red, comunicación personal, 07/03/2016).

El trabajo de proximidad es además entendido por las personas entrevistadas como poseedor de características singulares y por esto mismo es necesario. La singularidad se deriva de los aspectos recientemente mencionados y se destaca la ausencia de este abordaje en el resto de la institucionalidad pública. También se considera necesario, puesto que es el medio en el que podrían alcanzarse los objetivos mencionados.

Solamente con la proximidad vos podes entender o ver a la familia. Si esto lo trabajaras desde una institución donde la familia tuviese que trasladarse, hay muchas cosas que te perdés. El hecho de recorrer el barrio, las instituciones, de ser parte de ellos, de acompañar, le va dando a la familia otra visión. A veces se cuestiona que la familia no va al sistema de salud $y$ al sistema educativo y después cuando vos acompañas a la familia, entendés por qué muchas veces se alejan de ese servicio, no te digo que se la discrimina, pero sí se la estigmatiza como que no puede, que no se comprometió y entendés por qué las familias no logran ciertas cosas (Operadora Cercanías, comunicación personal, 03/03/2016).

Somos un nexo entre las familias y las instituciones, a veces pasa que las instituciones no salen y necesitás atraer a la población. El trabajo de cercanía está pensado para trabajar cosas que tal vez no se trabajan en las instituciones porque los tiempos no están para que se los dediquen a las familias. (...) esto permite un tiempo más distendido, con un vínculo más cercano, con cierta comodidad, porque estás en su casa y se pueden trabajar más aspectos que tal vez no se pueden trabajar en otros ámbitos (Operadora UCC, comunicación personal, 30/03/2016).
Asimismo, el trabajo de proximidad permitiría destacar las particularidades de los territorios donde se implementan los programas, así como las de sus receptores. Esto aparece de manera importante en el discurso de las entrevistas.

En teoría [la proximidad] está bien pensada y los inconvenientes vienen en la implementación. Te hace conocer en detalle las particularidades de cada una de las familias. Que son esas particularidades las que las dejan por fuera. Por más que tengan cerquita a las redes, son lo que las dejan por fuera (Operador Cercanías, comunicación personal, 05/04/2016).

En los casos que el entramado institucional es nombrado en las entrevistas, no surge un trabajo relevante en este sentido. Cuando las instituciones aparecen, están asociadas a articular y coordinar, pero no surgen en estas las ideas de acuerdos o cambios.

\section{TENSIONES QUE SURGEN EN EL TRABAJO DE PROXIMIDAD}

Durante la implementación de los programas, la realidad toma distancia con lo señalado y las opciones innovadoras encuentran límites no considerados en el diseño. Básicamente, se observan dos procesos: se amplía el espacio del trabajo de proximidad y eso agudiza los límites de este abordaje.

Como fuera indicado, los programas plantean como novedades el trabajo de proximidad, pero también la intersectorialidad. La apuesta es que el diseño interinstitucional y la gestión compartida entre diversas instituciones, habilite un acceso más fácil y con mayor cantidad de recursos. Las personas entrevistadas son contundentes en indicar que eso no ha ocurrido y entonces -y más allá del diseñola práctica de los programas tiende a quedar básicamente centrada en el trabajo de proximidad.

Se produce entonces una ampliación y lo que en este texto se considera, sobrevaloración del trabajo de proximidad. Los programas que se dirigen a la población en situaciones más extremas, 
logran movilizar muy escasos recursos nuevos y así, la baja materialidad de la política asistencial se vuelve más cruda. Esto lleva a que "el" recurso en muchos casos sea el operador y a una sobreestimación de la escucha y la palabra.

A veces estas mujeres lo único que necesitan es ser escuchadas, más allá de todas las carencias que puedan tener, lo que realmente necesitan es alguien que las trate como personas. Ese es el gran fruto del trabajo en cercanía, del acompañamiento. Cuando hacés la evaluación remarcan eso 'me escucharon, me apoyaron, me acompañaron, no me decían que hago todo mal' (Supervisora Ucc, comunicación personal, 28/03/2016).

Esto no supone desconocer la importancia que tienen para todos la escucha y la palabra, en cambio implica problematizar sobre sus posibilidades y límites, en contextos de extrema pobreza que permanecen casi incambiados en el marco de estos programas. El otro proceso observado es un entrampamiento y aquello que se configura como tan particular y original, parece volverse sobre los programas. Algunos entrevistados reconocen los límites de estas intervenciones sin que se modifiquen aspectos de la vida de quienes participan de los programas. Pero aún así tiende a prevalecer el trabajo de proximidad $y$ de nuevo, dificultades para alcanzar los objetivos.

Los programas quedan atrapados en la originalidad y no logra quedar claro como se espera transitar desde un dispositivo tan personalizado y singular, a otros (mundo del trabajo y resto de las prestaciones públicas) que implican siempre niveles de generalidad $y$ homogeneidad. Sobre todo considerando que en muchos casos, fueron instituciones en las cuales ya estuvieron participando. Resulta inevitable preguntarse sobre las propiedades que se le atribuyen a la proximidad y que permitirían - solamente con base en el trabajo con los participantes - modificar un vínculo que ya tuvo dificultades.

Estos gurises todavía no tienen algunas estrategias necesarias para poder llegar a esas instituciones universales, que en el mejor de los casos los van a tratar a todos igual $y$ precisan de estrategias que se adapten un poco más a sus necesidades $y$ sus posibilidades. Necesitan de alguien que pueda pensar una estrategia para él o para ella (Supervisora Jóvenes en Red, comunicación personal, 17/03/2016).

A esas poblaciones les cuesta mucho llegar a las policlínicas y ni que hablar de la marginación socioeconómica que también es un obstáculo para que puedan acceder a los servicios, entonces creo que este dispositivo también es una manera de presentar nuevas experiencias a las familias con equipos del Estado, a partir del vínculo de confianza puede generarse una experiencia positiva y que puede sanar otras (Operadora UCC, comunicación personal, 18/03/2016).

La apuesta afectiva que la proximidad supone también genera complejidades. Se trata de un vínculo que muchas veces termina siendo poco claro para quienes participan en los programas y que además, es transitorio.

Porque ese tiempo en la familia genera en el otro un ‘qué sos? ¿Sos mi amigo? ustedes son los que tengo en mis números favoritos en el celular'. A veces te preguntan '¿vos querés ser mi amiga? Porque vos siempre me escuchás y me das para adelante'. Ahí vos explicas 'yo no soy tu amiga porque viste que yo estoy de pasada'. ¿Cómo respondés a esa situación? No hay librito que te diga cómo responder (Operadora Cercanías, comunicación personal, 18/03/2016).

Los programas aparecen -nuevamente - envueltos en sus propias apuestas y aquello que sostiene el trabajo, es justamente lo que se terminará y que no parece trasladable a otra institución. Así, se reiteran los relatos que indican la permanencia de un vínculo aún después de finalizado el plazo de trabajo definido. 
Se supone que colaboramos en la construcción de esas fortalezas necesarias para sostener su propio camino y proceso, a veces eso no sucede. Si bien la idea del programa es esa, la realidad y lo que pasa, es que hay gurises que generan una referencia muy fuerte, siguen viniendo $y$ nosotros seguimos acompañando pero desde otro lugar (Coordinador Jóvenes en Red, comunicación personal, 09/03/2016).

Entonces, se trata de un trabajo particular y singular, que no se desarrollaría en otras instituciones, pero que tampoco se promueve en ellas, en tanto, esta realidad institucional aparece solo como un dato. Incluso, parece observarse en algunos casos, una suerte de traslado de responsabilidad por los receptores, a los programas de proximidad. Este aspecto, parece potenciado por el ya referido a la excepcionalidad de la proximidad.

En el "entendible" rescate del trabajo que se realiza, se concibe como algo tan singular que parecería promover una suerte de especialización, de manera que los programas de proximidad terminarían quedando como los únicos que llevan $-y$ pueden llevar - adelante este abordaje y opera una reducción del trabajo que 'puertas afuera' realizan otras instituciones.

En la Escuela, dicen 'ah, estos gurises son de ETAF', $y$ no, no son nuestros $y$ segundo que es una intervención que se terminó. En general si hay un ETAF las instituciones colocan la responsabilidad en el ETAF, de lo que se hace, de lo que no se hace. Es un trabajo constante, no es nuestra familia, es una familia que circula en el territorio en el que las redes deben fluir. Y es una intervención acotada en el tiempo (Operadora Cercanías, comunicación personal, 05/04/2016).

En muchos casos, se instala una pregunta por el sentido del trabajo, básicamente derivado de movilizar asuntos para los cuales después no habrá respuesta. En cambio, se observa menos la pregunta sobre el qué hacer ante el no acceso a derechos.
Nos ha pasado de movilizar a una mamá, llamando previamente $y$ haciendo las coordinaciones, donde la madre hace todo el proceso para salir de su casa finalmente y cuando llegamos nos dicen 'no tengo ningún lugar', y la mujer ya está con el bolso y todo ahí. Quedás como en una situación de ¿qué hice? Hay que ver bien qué movilizas porque después no sabes si vas a tener una respuesta para eso (Operadora UCC, comunicación personal, 30/03/2016).

Las formas que adopta la proximidad en los términos señalados, sumado a las condiciones de trabajo para quienes allí operan, hacen que sea vivido $-y$ así es expresado- como un trabajo desgastante, que no puede sostenerse toda la vida, en el cual, los equipos deben ser cuidados. Lo planteado lleva a que existan grandes dificultades para el cumplimiento de los objetivos. Como "respuesta" a esta situación se observan dos movimientos: por un lado, la sobrevaloración del espacio del encuentro con el otro $y$ un énfasis -que se considera excesivo- en el trabajo sobre aspectos subjetivos; por el otro, una necesidad de ajustar los objetivos. En muchos casos esto aparece explícitamente planteado, en otros es visualizable en la demanda de considerar los procesos además de los resultados, lo que supone valorizar los trayectos realizados por los receptores.

Es necesario bajar un poco los objetivos, hay objetivos como la revinculación educativa y la laboral que trascienden lo que podemos hacer, porque están determinadas por otras cosas que son ajenas a nosotros o a lo que puede llegar a ser nuestro trabajo (Supervisora Jóvenes en Red, comunicación personal, 17/03/2016).

[El registro] es cuantitativo no tiene mucho espacio para lo cualitativo, que es lo que a veces cuestionamos, porque hay cosas que quedan de lado y no se ven tanto los procesos porque si medís: se logró, no se logró o se logró parcial, se pierden los procesos de que hay cosas 
que sí, que las familias van logrando, pero no llega a ser ni un logro parcial (Operadora Cercanías, comunicación personal, 03/03/2016).

Lo indicado no implica desconocer la relevancia de los procesos y solamente valorar los resultados, pero sí supone advertir que la preocupación por los primeros suele ser colocada ante el no cumplimiento de los objetivos; sobre todo, llamar la atención sobre la ausencia de la pregunta por dónde y a partir de qué dispositivos esos objetivos serán alcanzados. Si no son viabilizados desde estos programas, ¿desde dónde lo serán? Por tanto, surge la duda en relación a las posibilidades políticas que en algún caso ayudarían a promover estos programas.

La proximidad es el insumo que permite que determinados derechos tengan la oportunidad de ser colocados. La proximidad lo que permite es el acercamiento al derecho vulnerado, a la colocación de esto como denuncia y revertir un escenario de vulneración de derechos (Operadora Cercanías, comunicación personal, 18/03/2016).

Ante esta perspectiva, además de preguntas en relación al sentido de activar demandas para las que no se tienen respuestas, resulta inevitable cuestionarse si existen mecanismos para mostrar esta realidad. Finalmente, esta posibilidad política no es lo que se observa, en cambio, la proximidad parece quedar atrapada en ella misma.

\section{SÍNTESIS Y CONCLUSIONES}

Este trabajo tomó como objeto de análisis los programas de proximidad "Cercanías", "Jóvenes en Red" y "Uruguay Crece Contigo", que desde el año 2012 se han implementado en Uruguay. Constituyen la última innovación y apuesta a la atención a las situaciones de extrema pobreza. Específicamente, estuvo centrado en visualizar la forma en que aparece conceptualizado el trabajo de proximidad y sus justificaciones, en el diseño de los programas y en el discurso de quienes allí trabajan. Asimismo, procuró identificar tensiones que se visualizan durante su implementación.

Se observa que el trabajo de proximidad está - tanto en el diseño como en los discursos- bajamente conceptualizado y justificado, sobre todo, dialogando poco con la realidad en la que se desarrolla. Las observaciones a este, no suponen no valorar la intención de que el acceso a las políticas y programas sociales sea efectivamente real $y$ en condiciones de calidad para la población beneficiaria. En cambio, implica advertir que la forma en que se desarrolla (lo que se dice y lo que silencia), termina generado resultados socialmente complejos, expresados en una suerte de "enojo social" con las personas en condición de pobreza y la asistencia, lo que supone básicamente la desresponsabilización colectiva por la existencia de unos y otros.

Los diagnósticos que sustentan los programas de proximidad son poco claros y de similar manera a las conceptualizaciones que fueran presentadas sobre estos, parten de una serie de afirmaciones que no son problematizadas. En este sentido, no se avanza más allá de la constatación de que hay población que no accede a programas sociales $y$ tampoco de lo que supone la multidimensionalidad de la pobreza y las respuestas integrales a esta.

En relación con el último aspecto, es importante advertir sobre un proceso de "esencialización" de la multidimensionalidad, en relación con la pobreza. Por supuesto que esta no puede ser reducida a una cuestión de ingresos, suponiendo también otros aspectos relevantes. Pero la multidimensionalidad no es un elemento que caracterice exclusivamente a esta condición, sino al conjunto de la realidad social. La pobreza es tan multidimensional como lo es la riqueza que además de implicar ingresos, supone una específica inserción en el mundo del trabajo, una determinada territorialidad en la que se habita $y$ un particular acceso a la educación, la salud, la vivienda, la cultura y el poder. Lo que sí es específico de esta es el desarrollo de la vida en un escenario donde hay derechos a los que no se acceden o se hace de manera incompleta $y$ desigual. 
No poner el foco en el último aspecto y sí hacerlo en lo "multi" de las dimensiones, habilita a pensar en la integralidad como la respuesta. Pero se trata en realidad, de una respuesta casi vacía dado que se produce un segundo movimiento. La integralidad no opera promoviendo acciones que permitan responder a esos derechos a los que no se accede, sino que se la "traduce" a la forma en que las prestaciones operan, entonces las preocupaciones por la articulación y la coordinación se instalan y pasan a formar parte de los programas de proximidad.

Asimismo, no parece problematizarse el hecho que los discursos sobre integralidad se desarrollan básicamente en relación con las personas pobres y la pobreza, que son escasamente identificables en políticas sociales orientadas a otros sectores de la población. Tampoco el hecho que los espacios de articulación se promueven fundamentalmente en los territorios más pobres y en torno a estos. El problema central no es la escasa coordinación, sino que la pobreza permanece y más que esta, la desigualdad.

Vinculado a lo anterior, otro aspecto que también llama la atención es la preocupación que surge en muchas entrevistas por la dependencia de los receptores y la importancia de construir autonomía. Se trata de una inquietud que no avanza ni problematiza las condiciones ni las posibilidades bajo las cuales esa autonomía se vuelve posible. Esta supone, necesariamente, el acceso a la protección social, algo que los programas de proximidad no logran viabilizar por sí mismos, ni en su intención de vincular con otras intervenciones públicas y el mercado de trabajo. Así, programas dirigidos a la extrema pobreza, aparecen preocupados por la autonomía, pero se sustentan básicamente en la palabra, entre técnicos e instituciones, sobre todo, entre operadores $y$ receptores.

En sintonía con lo presentado sobre los programas, otro aspecto que surge en el discurso de las personas entrevistadas $-y$ sobre el cual quiere advertirse-, es la insistencia en las particularidades de los receptores de los programas. La posibilidad de identificar y trabajar sobre la singularidad es fuertemente destacado. El señalamiento y preocupación por este aspecto, no supone desconocer las singularidades de los sujetos, pero sí indicar que no se considera que este allí el punto central de respuesta a la pobreza. La exacerbación del discurso de las diferencias $y$ particularidades en la pobreza, termina contribuyendo a alejar la preocupación por los componentes generales de la misma y aquellos que la explican. Las dificultades para avanzar en la reducción sostenible de la pobreza están sustantivamente menos colocadas en no dar suficientemente cuenta de lo diverso, que en no hacerlo de lo general y que iguala a las personas pobres.

Tampoco se observa una problematización sobre los objetivos no alcanzados y/o las demandas que no se logran atender por los programas. El trabajo de proximidad se configura como un nuevo testigo de las trágicas condiciones en que continúan viviendo muchos niños, niñas, jóvenes, mujeres, familias. Pero este aspecto, que debería ingresar en el debate público, en términos de los resultados sociales no alcanzados, no lo hace o lo hace muy parcialmente.

Los programas de proximidad deben ser puestos en diálogo con la realidad de la que son parte. Esto implica reconocer la mejora en varios indicadores sociales, pero también la persistencia de la desigualdad. El discurso gubernamental solamente trae en el discurso los indicadores sociales en los que ha habido mejoras (baja en el desempleo y la informalidad, aumento del gasto público, reducción de la pobreza y la indigencia) pero no expone las diversas expresiones de la desigualdad que persisten, en el mercado de trabajo, en las condiciones y características de los distintos territorios, en la calidad de las prestaciones en la educación y la salud, en el acceso a la vivienda, infraestructura y el cuidado, entre otros. Insistir en los primeros desconociendo los segundos, sumado al discurso del gasto en política asistencial, solo contribuye a reforzar la idea de que la pobreza es un asunto individual y derivado del mérito personal, diluyendo la responsabilidad colectiva.

Es la desigualdad - expresada, entre otros, en el mercado de trabajo y en los servicios públicos-y no las circunstancias o 
características particulares, lo que explican la pobreza y la política asistencial. Colocarlo en los sujetos es históricamente el camino más fácil, pero también, el más peligroso para los sujetos particulares y para el colectivo social.

\section{REFERENCIAS}

Arias, A. (2012). Pobreza y modelos de intervención. Aportes para la superación del modelo de asistencia y promoción. Buenos Aires: Espacio Editorial.

Cecchini, S y Atuesta, B. (2017). Programas de transferencias condicionadas en América Latina y el Caribe. Tendencias de cobertura e inversión. Santiago: CEPAL.

Cercanías. (2015). Modelo de Atención. Montevideo: MIDES. Recuperado de www. mides.gub.uy

Filgueira, F. (2015). Modelos de desarrollo, matriz del Estado social y herramientas de las políticas sociales latinoamericanas. En S. Cecchini; F. Filgueira; R. Martínez; C. Rossel (editores). Instrumentos de protección social. Caminos latinoamericanos hacia la universalización (pp. 49-84). Santiago: CEPAL.

Jara, P. y Sorio, R. (2013). Análisis de modalidades de acompañamiento familiar en programas de apoyo a poblaciones vulnerables o en situación de pobreza. BID. Recuperado de www.publications.iadb.org

Jara, P., Barriga, P., Villafuerte, A. y González Acero, C. (2013). Modalidades de acompañamiento familiar en los servicios sociales. Memoria del Diálogo Regional de Protección Social y Salud. BID. Recuperado de www.publications.iadb.org Martínez Franzoni, J., y Sánchez-Ancochea, D. (2016). Regímenes de Bienestar en América Latina: tensiones entre universalización y segmentación. En E. del
Pino y J. Rubio Lara (directoras). Los Estados de Bienestar en la encrucijada. Politicas Sociales en perspectiva comparada (pp. 200-220). Madrid: Tecnos.

Ministerio de Desarrollo Social-mides. (2010). La oferta pública social en el Uruguay 2005-2009. Informe final del convenio con el ICP/FCS-UDELAR. Montevideo: MIDES.

Ministerio de Desarrollo Social-MIDEs (Sin fecha a). Matriz de Protección Social del Uruguay/Organismos Nacionales. Relevamiento 2011/2012. Montevideo, mimeo.

Ministerio de Desarrollo Social -MIDEs (Sin fecha b). Matriz de Protección Social del Uruguay/Organismos Nacionales. Relevamiento 2014/2015. Montevideo, mimeo.

MIDES, MEC, MTSS, MDN, MDYT, ANEP/UTU, INAU (2012). Programa Jóvenes en Red. Propuesta de elaboración interinstitucional. Monte-video, mimeo.

Mides, inau, anep, asse, mVotma (2012). Cercanías. Estrategia Nacional de Fortalecimiento de las Capacidades Familiares. Montevideo, mimeo.

Soldano, D. y Andrenacci, L. (2005). Aproximación a las teorías de la política social a partir del caso argentino. En L. Andrenacci (comp). Problemas de politica social en la Argentina contemporánea (pp. 17-79). Buenos Aires: UnGs-Prometeo Libros.

Uruguay Crece Contigo-UCC (Sin fecha a). Programa Uruguay Crece Contigo. Montevideo.

Uruguay Crece Contigo-Ucc (Sin fecha b). Políticas de Primera Infancia. Uruguay Crece Contigo. Montevideo, mimeo.

Fecha de ingreso: 04/09/2018 Fecha de aprobación: 22/03/2019 
